\title{
Continuity of Multilinear Operator on Normed Linear Spaces
}

\author{
Kazuhisa Nakasho \\ Yamaguchi University \\ Yamaguchi, Japan
}

\author{
Yasunari Shidama \\ Shinshu University \\ Nagano, Japan
}

\begin{abstract}
Summary. In this article, various definitions of contuity of multilinear operators on normed linear spaces are discussed in the Mizar formalism [4], 1] and 2]. In the first chapter, several basic theorems are prepared to handle the norm of the multilinear operator, and then it is formalized that the linear space of bounded multilinear operators is a complete Banach space.

In the last chapter, the continuity of the multilinear operator on finite normed spaces is addressed. Especially, it is formalized that the continuity at the origin can be extended to the continuity at every point in its whole domain. We referred to [5, [1], 8, ,9] in this formalization.
\end{abstract}

\section{MSC: 46-00 47A07 47A30 68T99 03B35}

Keywords: Lipschitz continuity; bounded linear operators; multilinear operators; Banach space

MML identifier: LOPBAN11, version: 8.1.09 5.54.1341

\section{Completeness of the Space of Multilinear Operators}

Now we state the propositions:

(1) Let us consider a natural number $n$, and a real number $r$. Suppose $0<r$. Then there exists a real number $s$ such that

(i) $0<s<r$, and

(ii) $\sqrt{s \cdot s \cdot n}<r$. 
(2) Let us consider finite sequences $R_{1}, R_{2}$ of elements of $\mathbb{R}$, natural numbers $n, i$, and a real number $r$. Suppose $i \in \operatorname{dom} R_{1}$ and $R_{1}=n \mapsto$ (1 qua real number) and $R_{2}=R_{1}+\cdot(i, r)$. Then $\prod R_{2}=r$.

(3) Let us consider a finite sequence $F$ of elements of $\mathbb{R}$. Suppose for every element $k$ of $\mathbb{N}$ such that $k \in \operatorname{dom} F$ holds $0 \leqslant F(k)$. Then $0 \leqslant \prod F$.

Proof: Define $\mathcal{P}$ [natural number] $\equiv$ for every finite sequence $F$ of elements of $\mathbb{R}$ such that for every element $k$ of $\mathbb{N}$ such that $k \in \operatorname{dom} F$ holds $0 \leqslant F(k)$ and len $F=\$_{1}$ holds $0 \leqslant \prod F$. For every natural number $n$ such that $\mathcal{P}[n]$ holds $\mathcal{P}[n+1]$. $\mathcal{P}[0]$. For every natural number $n, \mathcal{P}[n]$.

From now on $X, G$ denote real norm space sequences, $Y$ denotes a real normed space, and $f$ denotes a multilinear operator from $X$ into $Y$.

Now we state the propositions:

(4) $\operatorname{dom} \bar{X}=\operatorname{dom} X$.

(5) Let us consider an element $z$ of $\prod X$. If $z=0 \prod_{X}$, then for every element $i$ of $\operatorname{dom} X, z(i)=0_{X(i)}$. The theorem is a consequence of (4).

(6) $f\left(0_{\prod_{X}}\right)=0_{Y}$. The theorem is a consequence of $(5)$.

(7) Let us consider a finite sequence $F$ of elements of $\mathbb{R}$. If for every element $i$ of $\operatorname{dom} F, F(i)>0$, then $\prod F>0$.

(8) Let us consider a real norm space sequence $X$, and a real normed space $Y$. Suppose $Y$ is complete. Let us consider a sequence $s_{1}$ of NormSpaceOfBoundedMultOpers $\operatorname{Na}_{\mathbb{R}}(X, Y)$. If $s_{1}$ is Cauchy sequence by norm, then $s_{1}$ is convergent.

Proof: Define $\mathcal{P}$ [set, set] $\equiv$ there exists a sequence $x_{1}$ of $Y$ such that for every natural number $n, x_{1}(n)=(\operatorname{PartFuncs}(v \operatorname{seq}(n), X, Y))\left(\$_{1}\right)$ and $x_{1}$ is convergent and $\$_{2}=\lim x_{1}$. For every element $x$ of $\prod X$, there exists an element $y$ of $Y$ such that $\mathcal{P}[x, y]$. Consider $f$ being a function from the carrier of $\prod X$ into the carrier of $Y$ such that for every element $x$ of $\prod X, \mathcal{P}[x, f(x)]$. Reconsider $t_{1}=f$ as a function from $\prod X$ into $Y$. For every point $u$ of $\prod X$ and for every element $i$ of $\operatorname{dom} X$ and for every point $x$ of $X(i)$, there exists a sequence $x_{2}$ of $Y$ such that for every natural number $n, x_{2}(n)=((\operatorname{PartFuncs}(v \operatorname{seq}(n), X, Y)) \cdot(\operatorname{reproj}(i, u)))(x)$ and $x_{2}$ is convergent and $\left(t_{1} \cdot(\operatorname{reproj}(i, u))\right)(x)=\lim x_{2} \cdot t_{1}$ is Lipschitzian by [10, $(20)$.

For every real number $e$ such that $e>0$ there exists a natural number $k$ such that for every natural number $n$ such that $n \geqslant k$ for every point $x$ of $\prod X,\left\|(\operatorname{PartFuncs}(\operatorname{vseq}(n), X, Y))(x)-t_{1}(x)\right\| \leqslant e \cdot($ NrProduct $x)$. Reconsider $t_{2}=t_{1}$ as a point of NormSpaceOfBoundedMultOpers $\mathbb{R}_{\mathbb{R}}(X, Y)$. For every real number $e$ such that $e>0$ there exists a natural number $k$ such that for every natural number $n$ such that $n \geqslant k$ holds $\| v \operatorname{seq}(n)-$ 
$t_{2} \| \leqslant e$. For every real number $e$ such that $e>0$ there exists a natural number $m$ such that for every natural number $n$ such that $n \geqslant m$ holds $\left\|v \operatorname{seq}(n)-t_{2}\right\|<e$.

(9) Let us consider a real norm space sequence $X$, and a real Banach space $Y$. Then NormSpaceOfBoundedMultOpers $\mathbb{R}_{\mathbb{R}}(X, Y)$ is a real Banach space. The theorem is a consequence of (8).

Let $X$ be a real norm space sequence and $Y$ be a real Banach space. One can check that NormSpaceOfBoundedMultOpers $\mathbb{R}_{\mathbb{R}}(X, Y)$ is complete.

\section{Equivalence of Continuity Definitions of Multilinear OPERATORS}

Now we state the propositions:

(10) Let us consider a natural number $n$, an element $F$ of $\mathcal{R}^{n}$, and a real number $s$. Suppose for every natural number $i$ such that $i \in \operatorname{dom} F$ holds $0 \leqslant F(i) \leqslant s$. Then $|F| \leqslant \sqrt{s \cdot s \cdot(\operatorname{len} F)}$.

Proof: Set $G=\operatorname{len} F \mapsto s$. Reconsider $F_{0}=F$ as an element of $\mathbb{R}^{\text {len } F}$. For every natural number $j$ such that $j \in$ Seg len $F_{0}$ holds $\left({ }^{2} F_{0}\right)(j) \leqslant\left({ }^{2} G\right)(j)$.

(11) Let us consider a real norm space sequence $X$, a real normed space $Y$, a multilinear operator $f$ from $X$ into $Y$, and a real number $K$. Suppose $0 \leqslant K$ and for every point $x$ of $\prod X,\|f(x)\| \leqslant K \cdot(\operatorname{NrProduct} x)$. Let us consider points $v_{0}, v_{1}$ of $\prod X$, finite sequences $C_{0}, C_{1}$, and an element $i$ of $\operatorname{dom} X$. Suppose $C_{0}=v_{0}$ and $C_{1}=v_{1}$ and $\left\|v_{1}-v_{0}\right\| \leqslant 1$ and for every element $j$ of $\operatorname{dom} X$ such that $i \neq j$ holds $C_{1}(j)=C_{0}(j)$. Then $\left\|f_{/ v_{1}}-f_{/ v_{0}}\right\| \leqslant\left(\left\|v_{0}\right\|+1\right)^{\operatorname{len} X} \cdot K \cdot\left\|\left(v_{1}-v_{0}\right)(i)\right\|$.

PROOF: For every object $x$ such that $x \in \operatorname{dom} v_{1} \operatorname{holds} v_{1}(x)=\left(\operatorname{reproj}\left(i, v_{0}\right)\right)$ $\left(v_{1}(i)\right)(x)$. Reconsider $v_{3}=\left(\operatorname{reproj}\left(i, v_{0}\right)\right)\left(v_{1}(i)-v_{0}(i)\right)$ as a point of $\prod X$. Reconsider $R_{1}=$ len $X \mapsto$ (1 qua real number) as a finite sequence of elements of $\mathbb{R}$. Reconsider $N_{1}=\left\|\left(v_{1}-v_{0}\right)(i)\right\|$ as an element of $\mathbb{R}$. Reconsider $R_{2}=R_{1}+\cdot\left(i, N_{1}\right)$ as a finite sequence of elements of $\mathbb{R}$. Reconsider $R_{3}=$ len $X \mapsto\left(\left\|v_{0}\right\|+1\right)$ as a finite sequence of elements of $\mathbb{R}$. Set $R_{4}=R_{2} \bullet R_{3} \cdot \prod R_{2}=\left\|\left(v_{1}-v_{0}\right)(i)\right\|$. Consider $N_{2}$ being a finite sequence of elements of $\mathbb{R}$ such that $\operatorname{dom} N_{2}=\operatorname{dom} X$ and for every element $i$ of $\operatorname{dom} X, N_{2}(i)=\left\|v_{3}(i)\right\|$ and NrProduct $v_{3}=\prod N_{2}$. For every element $k$ of $\mathbb{N}$ such that $k \in \operatorname{dom} N_{2}$ holds $N_{2}(k) \leqslant R_{4}(k)$ and $0 \leqslant N_{2}(k)$.

(12) Let us consider a real norm space sequence $X$, a real normed space $Y$, a multilinear operator $f$ from $X$ into $Y$, and a real number $K$. Suppose 
$0 \leqslant K$ and for every point $x$ of $\prod X,\|f(x)\| \leqslant K \cdot($ NrProduct $x)$. Let us consider a point $v_{0}$ of $\prod X$. Then there exists a real number $M$ such that

(i) $0 \leqslant M$, and

(ii) for every point $v_{1}$ of $\prod X$ such that $\left\|v_{1}-v_{0}\right\| \leqslant 1$ there exists a finite sequence $F$ of elements of $\mathbb{R}$ such that $\operatorname{dom} F=\operatorname{dom} X$ and $\| f_{/ v_{1}}-$ $f_{/ v_{0}} \| \leqslant M \cdot K \cdot\left(\sum F\right)$ and for every element $i$ of $\operatorname{dom} X, F(i)=$ $\left\|\left(v_{1}-v_{0}\right)(i)\right\|$.

Proof: Consider $g$ being a function such that $v_{0}=g$ and $\operatorname{dom} g=\operatorname{dom} \bar{X}$ and for every object $i$ such that $i \in \operatorname{dom} \bar{X}$ holds $g(i) \in \bar{X}(i)$. Reconsider $C_{0}=v_{0}$ as a finite sequence. Define $\mathcal{P}$ [natural number] $\equiv$ for every points $v_{0}, v_{1}$ of $\prod X$ for every finite sequences $C_{0}, C_{1}$ such that $\left\|v_{1}-v_{0}\right\| \leqslant$ 1 and $v_{0}=C_{0}$ and $v_{1}=C_{1}$ and $\$_{1} \leqslant \operatorname{len} X$ and $C_{1}$ 个(len $\left.X-^{\prime} \$_{1}\right)=$ $C_{0} \uparrow\left(\right.$ len $\left.X-{ }^{\prime} \$_{1}\right)$ there exists a finite sequence $F$ of elements of $\mathbb{R}$ such that $\operatorname{dom} F=\operatorname{Seg} \$_{1}$ and $\left\|f_{/ v_{1}}-f_{/ v_{0}}\right\| \leqslant\left(\left\|v_{0}\right\|+3\right)^{\operatorname{len} X} \cdot K \cdot\left(\sum F\right)$ and for every natural number $n$ such that $n \in \operatorname{Seg} \$_{1}$ there exists an element $i$ of $\operatorname{dom} X$ such that $i=\operatorname{len} X-{ }^{\prime} \$_{1}+n$ and $F(n)=\left\|\left(v_{1}-v_{0}\right)(i)\right\|$.

$\mathcal{P}[0]$. For every natural number $k$ such that $\mathcal{P}[k]$ holds $\mathcal{P}[k+1]$. For every natural number $n, \mathcal{P}[n]$. Consider $g$ being a function such that $v_{1}=g$ and $\operatorname{dom} g=\operatorname{dom} \bar{X}$ and for every object $i$ such that $i \in \operatorname{dom} \bar{X}$ holds $g(i) \in \bar{X}(i)$. Consider $F$ being a finite sequence of elements of $\mathbb{R}$ such that $\operatorname{dom} F=\operatorname{Seg}$ len $X$ and $\left\|f_{/ v_{1}}-f_{/ v_{0}}\right\| \leqslant\left(\left\|v_{0}\right\|+3\right)^{\text {len } X} \cdot K \cdot\left(\sum F\right)$ and for every natural number $n$ such that $n \in$ Seg len $X$ there exists an element $i$ of $\operatorname{dom} X$ such that $i=\operatorname{len} X-^{\prime} \operatorname{len} X+n$ and $F(n)=\left\|\left(v_{1}-v_{0}\right)(i)\right\|$. For every element $i$ of $\operatorname{dom} X, F(i)=\left\|\left(v_{1}-v_{0}\right)(i)\right\|$.

(13) Let us consider a point $x$ of $\prod X$, and a real number $r$. Suppose $0<r$. Then there exists a finite sequence $s$ of elements of $\mathbb{R}$ and there exists a non empty, non-empty finite sequence $Y$ such that $\operatorname{dom} s=\operatorname{dom} X$ and $\operatorname{dom} Y=\operatorname{dom} X$ and $\Pi Y \subseteq \operatorname{Ball}(x, r)$ and for every element $i$ of $\operatorname{dom} X$, $0<s(i)<r$ and $Y(i)=\operatorname{Ball}(x(i), s(i))$.

Proof: Consider $s_{0}$ being a real number such that $0<s_{0}<r$ and $\sqrt{s_{0} \cdot s_{0} \cdot(\text { len } X)}<r$. Set $C_{2}=\operatorname{len} X \mapsto s_{0}$. For every element $i$ of $\operatorname{dom} X$, $0<C_{2}(i)<r$. Define $\mathcal{P}$ [object, object] $\equiv$ there exists an element $i$ of $\operatorname{dom} X$ such that $\$_{1}=i$ and $\$_{2}=\operatorname{Ball}\left(x(i), C_{2}(i)\right)$. For every natural number $n$ such that $n \in$ Seg len $X$ there exists an object $d$ such that $\mathcal{P}[n, d]$. Consider $Y$ being a finite sequence such that $\operatorname{dom} Y=\operatorname{Seg} \operatorname{len} X$ and for every natural number $n$ such that $n \in \operatorname{Seg} \operatorname{len} X$ holds $\mathcal{P}[n, Y(n)] . \emptyset \notin$ $\operatorname{rng} Y$ by [6, (14)]. For every element $i$ of $\operatorname{dom} X, Y(i)=\operatorname{Ball}\left(x(i), C_{2}(i)\right)$. For every object $z$ such that $z \in \prod Y$ holds $z \in \operatorname{Ball}(x, r)$.

(14) Let us consider a real norm space sequence $X$, a real normed space $Y$, 
and a multilinear operator $f$ from $X$ into $Y$. Then

(i) $f$ is continuous on the carrier of $\prod X$ iff $f$ is continuous in $\prod_{X X}$, and

(ii) $f$ is continuous on the carrier of $\prod X$ iff $f$ is Lipschitzian.

Proof: $f_{/ 0} \prod_{x}=0_{Y}$. If $f$ is continuous in $0 \prod_{X}$, then $f$ is Lipschitzian by [7, (7)], (13), (4), (5). If $f$ is Lipschitzian, then $f$ is continuous on the carrier of $\prod X$ by (12), [3, (10)].

\section{REFERENCES}

[1] Grzegorz Bancerek, Czesław Byliński, Adam Grabowski, Artur Korniłowicz, Roman Matuszewski, Adam Naumowicz, Karol Pąk, and Josef Urban. Mizar: State-of-the-art and beyond. In Manfred Kerber, Jacques Carette, Cezary Kaliszyk, Florian Rabe, and Volker Sorge, editors, Intelligent Computer Mathematics, volume 9150 of Lecture Notes in Computer Science, pages 261-279. Springer International Publishing, 2015. ISBN 978-3319-20614-1. doi $10.1007 / 978-3-319-20615-8 \_17$.

[2] Grzegorz Bancerek, Czesław Byliński, Adam Grabowski, Artur Korniłowicz, Roman Matuszewski, Adam Naumowicz, and Karol Pack. The role of the Mizar Mathematical Library for interactive proof development in Mizar Journal of Automated Reasoning, 61(1):9-32, 2018. do1:10.1007/s10817-017-9440-6

[3] Noboru Endou, Yasunari Shidama, and Keiichi Miyajima. The product space of real normed spaces and its properties. Formalized Mathematics, 15(3):81-85, 2007. doi: $10.2478 /$ v10037-007-0010-y

[4] Adam Grabowski, Artur Korniłowicz, and Adam Naumowicz. Four decades of Mizar. Journal of Automated Reasoning, 55(3):191-198, 2015. doi $10.1007 / \mathrm{s} 10817-015-9345-1$

[5] Miyadera Isao. Functional Analysis. Riko-Gaku-Sya, 1972.

[6] Kazuhisa Nakasho, Yuichi Futa, and Yasunari Shidama. Implicit function theorem. Part I. Formalized Mathematics, 25(4):269-281, 2017. doi:10.1515/forma-2017-0026.

[7] Takava Nishivama, Keiji Ohkubo, and Yasunari Shidama. The continuous functions on normed linear spaces Formalized Mathematics, 12(3):269-275, 2004.

[8] Laurent Schwartz. Théorie des ensembles et topologie, tome 1. Analyse. Hermann, 1997.

[9] Laurent Schwartz. Calcul différentiel, tome 2. Analyse. Hermann, 1997.

[10] Yasunari Shidama. Banach space of bounded linear operators Formalized Mathematics, 12(1):39-48, 2004.

[11] Kosaku Yoshida. Functional Analysis. Springer, 1980.

Accepted February 27, 2019 